\title{
The Quality Gate and the Application of Momenta in Infinite Boundaries.
}

\author{
JOERG VOLKMANN \\ International Laboratory of Theoretical and \\ Mathematical Physics of Molecules and Crystals \\ Ufa Federal Research Centre, \\ Russian Academy of Sciences \\ Prospect Octyabrya, 71, Ufa, 450054 - RUSSIA \\ RUSSIA \\ Joerg_Volkmann@gmx.net \\ NAIL MIGRANOV \\ Department of Medical Physics \\ Bashkir State Medical University \\ Lenin Str. 3, Ufa, 450008, \\ RUSSIA \\ ufangm@yahoo.co.uk
}

\begin{abstract}
In this paper the concept of calculating the momenta in infinite boundaries is shown The Quality Gate in Science also plays a significant role. Based on Einstein's observation on ordinary diffusion, generalisations regarding time-fractional and space fractional derivatives are investigated. For both cases the fundamental solu- tion of a Cauchy problem with Dirac's delta function as initial distribution is determined. From these solutions the momenta are calculated. It could be shown that these momenta exist for the solution of the time fractional differential equation. The expressions for the momenta in infinite boundaries of the space fractional differential equations can be calculated and are only of academic interest because in most cases they do not exist. Furthermore is shown, that the momenta for the time fractional equation are always monotonically increasing functions. This draws our attention to the question how processes can be described in which the momenta are not monotonously increasing functions. Experiments with such behaviour can be found in practice.
\end{abstract}

Key-Words: Quality gate, diffusion, infinite momenta, variance theorem

Received: May 1, 2020. Revised: September 18, 2020. Accepted: September 26, 2020. Published: September 30, 2020.

\section{Introduction}

Since the famous work of Einstein $(* 13.03 .1879$ Ulm, + 18.04.1955 Princton, New Jersey, US) and Smoluchowski (*28.05.1872 Mödling, + 05.09.1917 Krakau) there is a relationship between the statistical quantity variance of a diffusion process and the diffusion constant $\mathrm{D}[1,2,3]$.

Starting point for this investigation is the diffusion equation of the form

$$
\frac{\partial \varrho}{\partial t}-D \frac{\partial^{2} \varrho}{\partial x^{2}}=s(t, x) .
$$

Here $D$ is the diffusion constant and the equation in general is formulated as an inhomogeneous one. An important role in this investigation plays the fundamental solution $F(t, x)$ which solves the related Cauchy problem

$$
\begin{aligned}
& \frac{\partial F(t, x)}{\partial t}-D \frac{\partial^{2} F(t, x)}{\partial x^{2}}=0, \\
& \left.F(t, x)\right|_{t \rightarrow 0}=\delta(x) .
\end{aligned}
$$

In this case $\delta(x)$ is the Dirac delta function. This solution is of the form

$$
F(t, x)=\frac{\exp \left(-\frac{x^{2}}{4 D t}\right)}{\sqrt{4 \pi D t}} .
$$

The comparison with the Gaussian distribution [4]

$$
f(t, x)=\frac{\exp \left(-\frac{(x-\mu)^{2}}{2 \sigma^{2}}\right)}{\sqrt{2 \pi \sigma^{2}}},
$$

where $\mu$ is the expectation value and $\sigma^{2}$ the variance of the distribution function leads to the statement

$$
\mu=0, \quad \sigma^{2}=2 D t .
$$

In [5] it was shown that this result can be calculated in a direct way. This brings the statistical momenta into focus.

In various experiments we can observe deviations from this linear behaviour (2) (see f. e. [6, 7, 8, 9, 10, 
11, 12]) which can be summarized by the application of a power law form (f. e. [13, 14, 15])

$$
\sigma^{2}=2 D_{\alpha} t^{\alpha}, \quad \alpha \in \mathbb{Q} .
$$

Metzler and Klafter used in [16] this relation to classify diffusion processes into subdiffusion, usual diffusion and superdiffusion.

But what does this situation mean in practice? In order to explain it in more detail the following fractional diffusion equation is considered:

$\frac{1}{\Gamma(1-\alpha)} \int_{0}^{t} \frac{1}{(t-\tau)^{-\alpha}} \frac{\partial T(\tau, x)}{\partial \tau} \mathrm{d} \tau=a \frac{\partial^{2} T(t, x)}{\partial x^{2}}$.

We are looking for a so called fundamental solution, which is given by

Definition 1. Consider the following Cauchyproblem:

$$
\begin{aligned}
& \frac{\partial^{\alpha} F(t, x)}{\partial t^{\alpha}}-D \frac{\partial^{2} F(t, x)}{\partial x^{2}}=0, \\
& \left.F(t, x)\right|_{t \rightarrow 0}=\delta(x),
\end{aligned}
$$

where $\delta(x)$ is Dirac's delta-function and

$$
\frac{\partial^{\alpha} F(t, x)}{\partial t^{\alpha}}=\frac{1}{\Gamma(1-\alpha)} \int_{0}^{t} \frac{1}{(t-\tau)^{-\alpha}} \frac{\partial T(\tau, x)}{\partial \tau} \mathrm{d} \tau .
$$

The solution of (甘) is called the fundamental solution of the Cauchy-problem (4).

In order to get the solution of (3) a Laplace transformation from $t$ to $p$ is applied. We find with $t>$ $0, \alpha>0$

$$
p^{-1+\alpha}(p \mathcal{L} T(p, x)-\delta(x))=a \frac{\partial^{2} \mathcal{L} T(p, x)}{\partial x^{2}} .
$$

Here $\mathcal{L} T(p, x)$ ist the Laplace transform of $T(t, x)$.

To this equation a Fourier-transformation from $x$ to $\omega$ is applied. This leads to

$$
p^{-1+\alpha}(-1+p \mathcal{F} \mathcal{L} T(p, \omega))=-a \omega^{2} \mathcal{F} \mathcal{L} T(p, x) .
$$

This equation can be solved for the Fourier-Laplacetransform $\mathcal{F} \mathcal{L} T(p, \omega)$ of $T(t, x)$. It is

$$
\mathcal{F} \mathcal{L} T(p, \omega)=\frac{p^{-1+\alpha}}{p^{\alpha}+a \omega^{2}} .
$$

This expression has to be transformed back into the original space. To do this first an inverse Fourier transformation from $\omega$ to $x$ has to be applied. This leads to

$$
\mathcal{L} T(p, \omega)=\frac{\exp \left(-\sqrt{\frac{p^{\alpha} x^{2}}{a}}\right) p^{\alpha-1}}{2 \sqrt{a} \sqrt{p^{\alpha}}}
$$

with $a>0$ and $\alpha>0$. In a second step the inverse Laplace transformation has to be applied. The result is

$$
\begin{aligned}
& T(t, x)= \\
& \frac{1}{\alpha x} \mathcal{H}_{1,1}^{1,0}\left[\frac{a^{-\frac{1}{\alpha}} x^{\frac{2}{\alpha}}}{t} \mid \begin{array}{c|c}
\{\} & \{\{1,1\}\} \\
\left\{\left\{1, \frac{2}{\alpha}\right\}\right\} & \{\}
\end{array}\right] .
\end{aligned}
$$

In order to simplify this solution a Mellin transformation from $a$ to $A$ has to be done. The result becomes with $\alpha>0$ and $t>0$

$$
\mathcal{M}_{a}^{A}[T(t, x)]=\frac{t^{-A \alpha} x^{-1+2 A} \Gamma(1-2 A)}{\Gamma(1-A \alpha)} .
$$

The back-transformation is

$$
\begin{aligned}
& T(t, x)= \\
& \frac{1}{x} \mathcal{H}_{1,1}^{1,0}\left[\frac{t^{-\alpha} x^{2}}{a} \mid \begin{array}{c|c}
\{\} & \{\{1, \alpha\}\} \\
\{\{1,2\}\} & \{\}
\end{array}\right] .
\end{aligned}
$$

It is obviously, that the case $\alpha=1$ in (3) leads to fundamental solution of the usual heat equation which is of the form (11). Taking (11), putting $\alpha \rightarrow 1$, making a Mellin transformation one arrives with $\alpha>0$ and $x>0$ at

$$
\mathcal{M}_{a}^{A}[T(t, x)]=\frac{\left(\frac{x^{2}}{t}\right)^{A} \Gamma(1-2 A)}{x \Gamma(1-A \alpha)} .
$$

The inverse Mellin transformation gives

$$
\left.T(t, x)\right|_{t \rightarrow 1}=\frac{\exp \left(-\frac{x^{2}}{4 a t}\right)}{2 \sqrt{a \pi t}} .
$$

To show the fulfillment of the initial condition we start with a Mellin transformation of (11) from $x$ to $X$ with $t>0, a>0, \alpha>0$. The result is

$$
\mathcal{M}_{a}^{A}[T(t, x)]=\frac{a^{\left(-\frac{1}{2}+\frac{X}{2}\right)} t^{-\frac{\alpha}{2}+\frac{X \alpha}{2}} \Gamma(x)}{2 \Gamma\left(1-\frac{\alpha}{2}+\frac{X \alpha}{2}\right)} .
$$

If $t \rightarrow 0$, one will find

$$
\left.\mathcal{M}_{a}^{A}[T(t, x)]\right|_{t \rightarrow 0}=\frac{a^{\left(-\frac{1}{2}+\frac{X}{2}\right)} 0^{-\frac{\alpha}{2}+\frac{X \alpha}{2}} \Gamma(x)}{2 \Gamma\left(1-\frac{\alpha}{2}+\frac{X \alpha}{2}\right)} .
$$

The inverse Mellin transformation leads to the Dirac's delta function which is the initial condition.

The check, wether the solution (11) is performed in Laplace Space, the Laplace-transformed solution

$$
\begin{aligned}
& \mathcal{L} T(p, x)= \\
& \frac{1}{2} a^{-\frac{1}{\alpha}-\frac{2+\alpha}{2 \alpha}} \exp \left(-\frac{p^{\frac{\alpha}{2}} x}{\sqrt{a}}\right) p^{\frac{1}{2}(-2+\alpha)} x^{-1+\frac{2}{\alpha}+\frac{-2+\alpha}{\alpha}}
\end{aligned}
$$


is inserted into the Laplace-transformed equation (5). The equation is thus fulfilled identically.

To ensure the quality of scientific results the quality gate is introduced in [5, 17] analogous to [18]. It says

\section{Quality gate [5, 17]}

In order to guarantee the quality of results, two or three different and independent ways have to be presented.

The quality gate is now applied in these calculations by reversing the inverse transformations. Starting with the inverse Laplace transformation follows in the Fourier space with $\alpha>0, \omega>0$

$$
\mathcal{F} T(t, \omega)=E_{\alpha, 1}\left[-a t^{\alpha} \omega^{2}\right],
$$

where

$$
E_{\alpha, \beta}[z]=\sum_{k=0}^{\infty} \frac{z^{k}}{\Gamma(\alpha k+\beta)}
$$

is the generalized Mittag-Leffler function with $\alpha, \beta \in$ $\mathbb{C}, \mathfrak{R}(\alpha)>0, \operatorname{Re}(\beta)>0, z \in \mathbb{C}$ (see f. e. [19]).

The application of the inverse Fouriertransformation leads to

$$
\begin{aligned}
& T(t, x)= \\
& t^{-\frac{\alpha}{2}} \mathcal{H}_{1,2}^{2,0}\left[\frac{t^{-\frac{\alpha}{2}} x}{2 \sqrt{a}} \mid \begin{array}{c|c}
\left\{\left\{0, \frac{1}{2}\right\},\left\{\frac{1}{2}, \frac{1}{2}\right\}\right\} & \left\{\left\{\frac{2-\alpha}{2}, \frac{\alpha}{2}\right\}\right\} \\
\{\}
\end{array}\right]
\end{aligned}
$$

In order to simplify this Fox-function a Mellintransformation from $a$ to $A$ is applied to (19):

$$
2 \pi \delta(A) T(t, x)=\frac{t^{-A \alpha} x^{-1+2 A} \Gamma(1.2 A)}{\Gamma(1-A \alpha)} .
$$

The inverse Mellin transform of (20) from $A$ to $a$ gives (11).

For the third calculation path according to the Quality Gate, the sequence of the integral transformations for the original equation is changed. Here the original equation is changed by a term responsible for the initial conditions. So the equation became

$$
\frac{1}{\Gamma(1-\alpha)} \int_{0}^{t}(t-\tau)^{\alpha} \frac{\partial T}{\partial \tau} \mathrm{d} \tau-\frac{\mathrm{t}^{-\alpha} \delta(\mathrm{x})}{\Gamma(1-\alpha)}=\mathrm{a} \frac{\partial^{2} \mathrm{~T}}{\partial \mathrm{x}^{2}} .
$$

Starting now with a Fourier-transformation, the result is

$$
\begin{aligned}
& \frac{1}{\Gamma(1-\alpha)} \int_{0}^{t}(t-\tau)^{\alpha} \frac{\partial \mathcal{F} T}{\partial \tau} \mathrm{d} \tau-\frac{\mathrm{t}^{-\alpha}}{\Gamma(1-\alpha)}= \\
& -a \omega^{2} \mid \mathcal{F} T(t, \omega) .
\end{aligned}
$$

If we now apply the Laplace transformation to this equation, neglecting the initial conditions which have already been considered, we arrive at

$$
-p^{-1+\alpha}+p^{\alpha} \mathcal{L F} T(p, \omega)=-a \omega^{2} \mathcal{L F} T(p, \omega) .
$$

Solving this equation for the Laplace-Fouriertransform, it is

$$
\mathcal{L F} T(p, \omega)=\frac{p^{-1+\alpha}}{p^{\alpha}+a \omega^{2}} .
$$

This solution is identical to (7). Thus three calculation paths have been shown according to the quality gate.

In the following the momenta of the solution (11) shall be calculated. In addition, the technical report [5, 17] introduced the concept of statistical momenta in infinite boundaries for evolutionary processes and discussed the example of heat conduction. The example of heat conduction has been investigated there. Starting point for the considerations was the

Definition 2. [4]

Let $X$ be a continuous random variable with the related density $f$ of a non-normalized distribution function $F(X)$. Then

$$
m_{i}=\int_{-\infty}^{\infty} x^{i} f(x) d x=<x^{i}, f(x)>
$$

is the $i$-th momentum and

$$
\mu_{i}=\int_{-\infty}^{\infty}\left(x-\frac{m_{1}}{m_{0}}\right)^{i} \frac{f(x)}{m_{0}} d x
$$

\section{the $i$-th normalized central momentum.}

In this definition the integration over the entire real axis with infinite boundaries has to be done.

Here the definition especially for the central momentum is different from the usual one which is

$$
\mu_{i}(X)=\int_{-\infty}^{+\infty}(x-\mu)^{i} f(x) d x
$$

with probability density function $f(x)$ which is the $i$ th momentum about the mean $\mu$ [20].

In the mathematical case a random variable $X$ is called continuous, if there exists a non-negative function $f(x)$, for which for real $x$ the relation

$$
F(x)=\int_{-\infty}^{x} f(t) d t
$$

is fullfilled. $F(x)$ is the distribution function of the random variable $X$. Furthermore is

$$
F(\infty)=\int_{-\infty}^{\infty} f(x) d x=1 .
$$

The last integral represents the norm and so the distribution function is normalized [21].

But if one deals with experimental data this condition is not self-evident. Experimental distribution 
functions of many kinds like the temperature distribution in a rod do not have this property. In order to apply the statistical theory of momenta this property has to be generated by calculating the norm and by normalizing the distribution function. The consequence is that the mean value in the central momentum has to be substituted by the normalized mean value $\frac{m_{1}}{m_{0}}$.

In order to establish the quality gate in the area of the calculation of momenta another definition concerning the momentum generating function is necessary:

Definition 3. [22]

A function $g(t)$ defined as follows

$$
\begin{aligned}
& g(t)=E\left(e^{t X}\right)=\sum_{j=0}^{\infty} \frac{m_{j} t^{j}}{j !}= \\
& E\left(\sum_{j=0}^{\infty} \frac{X^{j} t^{j}}{j !}\right)=\sum_{j=1}^{\infty} e^{t X_{j}} p\left(X_{j}\right)
\end{aligned}
$$

is called the momentum generating function.

By differentiating $g(t)$ with respect to $t$ and setting $t \rightarrow 0$,

$$
\begin{aligned}
& \left.\frac{d^{i}}{d t^{i}} g(t)\right|_{t \rightarrow 0}=g^{(i)}(0)= \\
& \left.\sum_{j=1}^{\infty} \frac{j ! m_{j} t^{j-i}}{(j-i) ! j !}\right|_{t \rightarrow 0}=m_{i}
\end{aligned}
$$

can be found. The momentum integral of definition 2 is setting in relation to the Mellin transformation, which is defined by

$$
\mathcal{M}_{x}^{z}[f(x)]=\int_{0}^{\infty} x^{z-1} f(x) \mathrm{dx} .
$$

Assuming symmetrical boundaries for the integration and a symmetrical distribution function the relationship between the momenta and the Mellin transformation of a function is

$$
\begin{aligned}
\left\langle x^{m}, f(x)\right\rangle & =2 \mathcal{M}_{x}^{m+1}[f(x)]= \\
& =2 \int_{0}^{\infty} x^{m+1-1} f(x) \mathrm{dx} .
\end{aligned}
$$

The application of this to the solution (11) gives

$$
\begin{aligned}
& \left\langle x^{m}, T(t, x)\right\rangle= \\
& 2 \mathcal{M}_{x}^{m+1}[ \\
& \frac{1}{x} \mathcal{H}_{1,1}^{1,0}\left[\frac{t^{-\alpha} x^{2}}{a} \mid \begin{array}{c|c}
\{\} & \{\{1, \alpha\}\} \\
\{1,2\}\} & \{\}
\end{array}\right] \\
& ] \\
= & \frac{a^{\frac{m}{2}} t^{\frac{m \alpha}{2}} \Gamma(1+m)}{\Gamma\left(1+\frac{m \alpha}{2}\right)} .
\end{aligned}
$$

$$
\begin{aligned}
& g(\tau)= \\
& \int_{-\infty}^{\infty} e^{\tau x} \frac{1}{x} \mathcal{H}_{1,1}^{1,0}\left[\frac{t^{-\alpha} x^{2}}{a} \mid \begin{array}{c|c}
\{\{\}\} & \{\{1, \alpha\}\} \\
\{\{1,2\}\} & \mid 30)
\end{array}\right] d x .
\end{aligned}
$$

To fulfill the quality gate the method with the momentum generating function is applied. In order to do this we have to solve the integral

This expression looks like a Laplace transformation. Assuming again a symmetrical distribution function with symmetrical boundaries follows

$$
\begin{aligned}
& g(\tau)= \\
& 2 \int_{0}^{\infty} e^{\tau x} \frac{1}{x} \mathcal{H}_{1,0}^{1,1}\left[\frac{t^{-\alpha} x^{2}}{a} \mid \begin{array}{c|c}
\{\{\}\} & \{\{1, \alpha\}\} \\
\{\{1,2\}\} & \{\}
\end{array}\right] d x= \\
& E_{\frac{\alpha}{2}}\left[-\sqrt{a} t^{\frac{\alpha}{2}} \tau^{2}\right]
\end{aligned}
$$

with the generalized Mittag -Leffler function (18). To calculate the variance this expression has to be differentiated twice with respect to $\tau$. This leads to

$$
\frac{d^{2} g}{d \tau^{2}}=a t^{\alpha}{ }_{1} \Psi_{1}\left[\begin{array}{c}
\{3,1\} \\
\left\{1+\alpha, \frac{\alpha}{2}\right\}
\end{array} \mid-\sqrt{a} t^{\frac{\alpha}{2}} \tau\right] .
$$

Here

$$
{ }_{p} \Psi_{q}\left[z \mid \begin{array}{c}
\left\{a_{p}, A_{p}\right\} \\
\left\{b_{q}, B_{q}\right\}
\end{array}\right]=\sum_{n=0}^{\infty} \frac{\prod_{j=1}^{p} \Gamma\left(a_{j}+n A_{j}\right)}{\prod_{j=1}^{q} \Gamma\left(b_{j}+n B_{j}\right)} \frac{z^{n}}{n !}
$$

with $a_{i}, b_{j} \in \mathbb{C}, A_{i}, B_{j} \in \mathbb{R}$ is called the Wright generalized hypergeometric function [19].

For the second momentum $m_{2}$ the parameter has to be zero. This gives

$$
\begin{aligned}
m_{2} & =\left.\frac{d^{2} g}{d \tau^{2}}\right|_{\tau \rightarrow 0} \\
& =\frac{2 a \tau^{\alpha}}{\Gamma(1+\alpha)} .
\end{aligned}
$$

Then the variance of the solution is

$$
\begin{aligned}
\sigma^{2} & =\frac{m_{2}}{m_{0}}-m_{1}^{2} m_{0}^{2} \\
& =a t^{\alpha}\left(\frac{2}{\Gamma(1+\alpha)}-\frac{1}{\Gamma\left(1+\frac{\alpha}{2}\right)^{2}}\right) .
\end{aligned}
$$

This presented concept is applied to the Lévy and the Cauchy distribution, and the application of the Quality Gate is also shown.

These distributions play a significant role for example in the theory of turbulence. Starting from various scaling laws Chen [23] motivated an equation of the form

$$
\frac{\partial P(t, x)}{\partial t}=(-\Delta)^{\frac{\alpha}{2}} P(t, x)
$$


with $0<\alpha \leq 2$. The case $\alpha=2$ should give the usual heat equation with the Gauss distribution as fundamental solution. But a closer look shows that the problem has to be formulated by

$$
\begin{aligned}
& \frac{\partial P(t, x)}{\partial t}=-D(-\Delta)^{\frac{\alpha}{2}} P(t, x), \\
& P(0, x)=\delta(x) .
\end{aligned}
$$

Here $\alpha$ and $D$ are constants with $0<\alpha \leq 2, D>0$ and $\delta(x)$ represents Dirac's delta function. Furthermore $(-\Delta)^{\frac{\alpha}{2}}$ is called Riesz-operator and is defined by

$$
\mathcal{F}_{x}^{\omega}\left((-\Delta)^{\frac{\alpha}{2}}\right) u=|\omega|^{\alpha} \mathcal{F}_{x}^{\omega}(u) .
$$

In order to solve equation (37) in a first step a Fouriertransform from $x$ to $\omega$ is applied to this equation. The result is

$$
\frac{\partial \mathcal{F}}{\partial t}(t, \omega)=-D \omega^{\alpha} \mathcal{F}(t, \omega),
$$

where $\mathcal{F}$ is the Fourier transform of $P$. Furthermore the initial condition has to be transformed, too. One can find

$$
P(0, x)=\delta(x) \quad \Leftrightarrow \quad \mathcal{F}(0, \omega)=1 .
$$

The second step now is to apply the Laplace transform from $t$ to $p$ to these equations. By taking into account the transformed initial condition follows

$$
\mathcal{L F}(p, \omega)=\frac{1}{p+D \omega^{\alpha}} .
$$

$\mathcal{L F}$ is the Laplace transform of the Fourier transform of $P$.

In order to fullfil the quality gate a second way can be chosen. It is to solve the ordinary differential equation (39) directly inclusive the initial condition (40). The solution is

$$
\mathcal{F}(t, \omega)=e^{-D t \omega^{\alpha}} .
$$

A Laplace transform from $t$ to $p$ leads to the solution (41). Both ways of calculation give identical results.

The result (41) now has to be expressed in the original coordinates. First an inverse Laplace transform has to be applied to (41). This leads to

$$
\mathcal{F}(t, \omega)=e^{-D t \omega^{\alpha}},
$$

which is identical to (42).

After this an inverse Fourier transform has to be applied. This leads to the solution

$$
\begin{aligned}
& P(t, x)=
\end{aligned}
$$

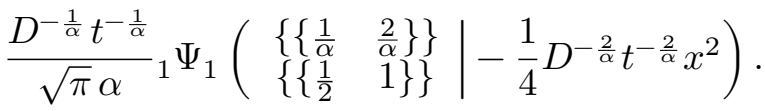

with the Wright hypergeometric function (33). This representation for the solution can be changed. In order to do this a Mellin transformation from $D$ to $\tilde{D}$ has to be done. This leads to

$$
\begin{aligned}
& \mathcal{M}_{D}^{\tilde{D}}[P(t, x)]= \\
& \frac{2^{-\tilde{D} \alpha} t^{-\tilde{D}} x^{-1+\tilde{D} \alpha} \Gamma(\tilde{D}) \Gamma\left(\frac{1}{2}-\frac{\tilde{D} \alpha}{2}\right)}{\sqrt{\pi} \Gamma\left(\frac{\tilde{D} \alpha}{2}\right)} .
\end{aligned}
$$

The back-transformation gives the solution of the problem (37) and can be regarded as [13]

$$
\begin{aligned}
& P(t, x)= \\
& \frac{1}{\sqrt{\pi}|x|} \mathcal{H}_{2,1}^{1,1}\left[\frac{2^{\alpha} D t}{|x|^{\alpha}} \mid \begin{array}{ccc}
\left\{\left\{\frac{1}{2}, \frac{\alpha}{2}\right\}\right\} & \left\{\left\{0, \frac{\alpha}{2}\right\}\right\} \\
\{\{0,1\}\} & \{\}
\end{array}\right] .
\end{aligned}
$$

Considering the case $\alpha=1$ the solution is

$$
\begin{aligned}
& P(t, x)= \\
& \frac{1}{\sqrt{\pi}|x|} \mathcal{H}_{2,1}^{1,1}\left[\frac{2 D t}{|x|} \mid \begin{array}{ccc}
\left\{\begin{array}{l}
\left.\left\{\frac{1}{2}, \frac{1}{2}\right\}\right\} \\
\{0,1\}\}
\end{array}\right. & \left\{\left\{0, \frac{1}{2}\right\}\right\} \\
\{0,1\}
\end{array}\right] .
\end{aligned}
$$

In order to simplify this expression a Mellintransformation from $D$ to $\tilde{D}$ is applied. The result is

$\mathcal{M}_{D}^{\tilde{D}}(P(t, x))=\frac{t^{-\tilde{D}} x^{-1+\tilde{D}} \Gamma\left(\frac{1}{2}-\frac{\tilde{D}}{2}\right) \Gamma\left(\frac{1}{2}+\frac{\tilde{D}}{2}\right)}{2 \pi}$.

The inverse Mellin-transformation gives

$$
P(t, x)=\frac{D t}{\pi\left(x^{2}+(D t)^{2}\right),}
$$

which is the Cauchy distribution function. In the case $\alpha=2$ the solution can be represented as

$$
\begin{aligned}
& P(t, x)= \\
& \frac{1}{\sqrt{\pi}|x|} \mathcal{H}_{2,1}^{1,1}\left[\frac{2^{2} D t}{|x|^{2}} \mid \begin{array}{c|c}
\left\{\left\{\frac{1}{2}, 1\right\}\right\} & \{\{0,1\}\} \\
\{\{0,1\}\} & \mid
\end{array}\right] .
\end{aligned}
$$

Applying to this solution the Mellin-transformation from $D$ to $\tilde{D}$ one finds

$$
\mathcal{M}_{D}^{\tilde{D}}(P(t, x))=\frac{4^{-\tilde{D}}\left(\frac{t}{x^{2}}\right)^{-\tilde{D}} \Gamma\left(\frac{1}{2}-\tilde{D}\right)}{\sqrt{\pi} \sqrt{x^{2}}},
$$

which leads after transforming this expression back to the original space via inverse Mellin-transformation to

$$
P(t, x)=\frac{1}{2 \sqrt{\pi D t}} \exp \left(-\frac{x^{2}}{4 D t}\right),
$$

which is the Gaussian distribution function. 
In general a Lévy-stable distribution is defined through its characteristic function of the probability density [16], that is, its Fourier transform

$$
\begin{aligned}
& p_{\alpha, \beta}(\omega ; \mu, \sigma)=\mathcal{F}_{x}^{\omega}\left(p_{\alpha, \beta}(x ; \mu, \sigma)\right) \\
= & \int_{-\infty}^{\infty} e^{i \omega x} p_{\alpha, \beta}(x ; \mu, \sigma) d x \\
= & \exp \left(i \mu \omega-\sigma^{\alpha}|\omega|^{\alpha}\left(1-i \beta \frac{\omega}{|\omega|} \bar{\omega}(\omega, \alpha)\right)\right)
\end{aligned}
$$

with

$$
\bar{\omega}=\left\{\begin{array}{ll}
\tan \left(\frac{\pi \alpha}{2}\right) & \text { if } \alpha \neq 1,0<\alpha<2 \\
-\frac{2}{\pi} \ln (|\omega|) & \text { if } \alpha=1
\end{array} .\right.
$$

The case $\alpha=2$ leads to the Gaussian distribution, $\alpha=1, \beta=0$ to the Cauchy distribution and $\alpha=$ $\frac{1}{2}, \beta=1$ to the Lévy-Smirnov distribution

$$
p_{\frac{1}{2}, 1}(x)=\left\{\begin{array}{l}
\frac{1}{\sqrt{2 \pi}} x^{-\frac{3}{2}} \exp \left(-\frac{1}{2 x}\right) \quad x \geq 0 \\
0 \quad x<0
\end{array}\right.
$$

\section{The Lévy Distribution}

In recent years, the Lévy distribution has been developed to study many physical phenomena, such as anomalous diffusion [6], power law response of material [24], aging in glassy systems [25], and so on [26]. This distribution has also been applied to Lévy glasses, to the turbulence in two spatial dimensions, and in pulsar scintillation [27], to the transport of carrier in disordered semiconductors [28]. The related random walk model is the so-called Lévy flight, named after the French mathematician Paul Pérre Lévy (1886 - 1971).

Here, the increments are described according to a heavily tailed probability distribution. This kind of models is adequate to the description of transport in heterogeneous catalysis, self diffusion in miscellaneous systems, reactions and transport in polymer systems under conformational motion, in transport processes in heterogenous rocks, and for description of behaviour of dynamical systems. Lévy's statistics were observed in hydrodynamic transport [29].

In the previous part we determine the fundamental solution of the Cauchy-problem (37) and found the Lévy distribution (49) for the case $\alpha=\frac{1}{2}, \beta=1$. This can be generalized to the form 50 from [30], where 49 can be recoverd by setting $\mu=0, \sigma=1$. The initial Lévy distribution can be regarded as [30]

$$
f(x)=\sqrt{\frac{\sigma}{2 \pi}} \frac{\exp \left(-\frac{\sigma}{2(x-\mu)}\right)}{(x-\mu)^{\frac{3}{2}}} .
$$

A more general form is [31], [32]

$$
f(x)=\frac{\sigma^{k}}{\Gamma(k)} \frac{\exp \left(-\frac{\sigma}{(x-\mu)}\right)}{(x-\mu)^{1+k}} .
$$

From this generalization 49 can be obtained by $\mu=$ $0, k=\frac{1}{2}, \sigma=\frac{1}{2}$.

\subsection{Discussion of Parameters}

The investigation starts with the Lévy distribution (50). Here can be found the two parameters $\sigma$ and $\mu$. As shown in figure (Fig. 1), $\sigma$ represents a scaling with the assumption $\sigma>0$.

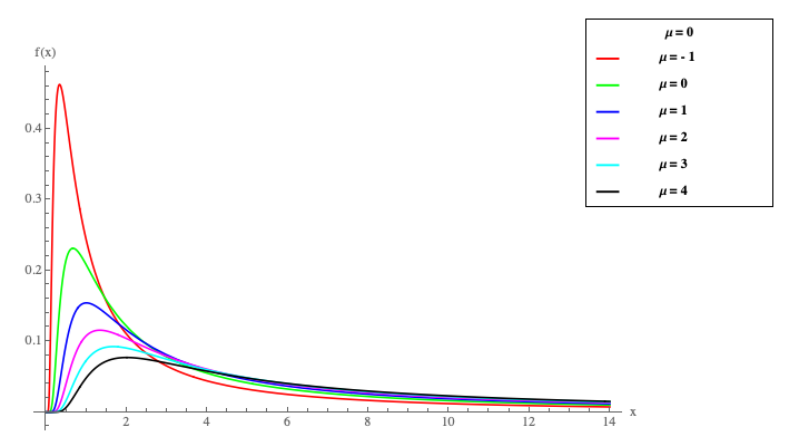

Figure 1:

The Lévy distribution (50) with fixed $\mu$ and varying $\sigma$.

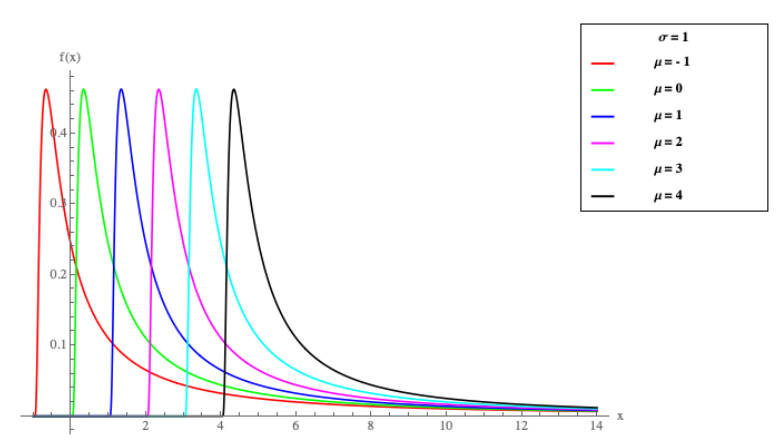

Figure 2:

The Lévy distribution (50) with fixed $\sigma$ and varying $\mu$.

Furthermore, figure (Fig.2) shows, that $\mu$ is responsible for a translation in $x$ direction with $\mu \in \mathbb{R}$. For the domain is valid: $D=\{x \mid x \geq \mu\}$, due to the square root in the denominator.

\subsection{Momenta with the momentum integral in infinite boundaries}

In analogy to the procedure which we have done with the time fractional diffusion equation, the momenta in infinite boundaries for the solutions should also be determined here in order to be able to judge the quality of diffusion processes.

Now, the momenta $m_{i}$ for the distribution (50) are calculated, whereby $i$ is their order. To use the integral representation, the boundaries have to be changed 
according to the area of the domain. The result is

$$
m_{i}=\int_{\mu}^{\infty} \sqrt{\frac{\sigma}{2 \pi}} \frac{x^{i} \exp \left(-\frac{\sigma}{2(x-\mu)}\right)}{(x-\mu)^{\frac{3}{2}}} \mathrm{~d} x .
$$

After the substitution $x-\mu=\xi$ can be found

$$
m_{i}=\sqrt{\frac{\sigma}{2 \pi}} \int_{0}^{\infty} \frac{(\xi+\mu)^{i} \exp \left(-\frac{\sigma}{2 \xi}\right)}{\xi^{\frac{3}{2}}} \mathrm{~d} \xi .
$$

The application of the binomial theorem leads to

$$
\begin{aligned}
& m_{i}= \\
& \sqrt{\frac{\sigma}{2 \pi}} \int_{0}^{\infty} \sum_{j=0}^{i}\left(\begin{array}{l}
i \\
j
\end{array}\right) \xi^{j} \mu^{i-j} \frac{\exp \left(-\frac{\sigma}{2 \xi}\right)}{\xi^{\frac{3}{2}}} \mathrm{~d} \xi .
\end{aligned}
$$

Here, the binomial sum is finite. Furthermore, summation and integration are swapped. The result is for the real part $\Re(j)<\frac{1}{2}$ by use of the confluent hypergeometric function

$$
\begin{aligned}
m_{i} & =\sum_{j=0}^{i} \frac{2^{-j} \mu^{i-j} \sigma^{j}\left(\begin{array}{c}
i \\
j
\end{array}\right) \Gamma\left(\frac{1}{2}-j\right)}{\sqrt{\pi}} \\
& =\mu^{i}{ }_{1} F_{1}\left(-i, \frac{1}{2}, \frac{\sigma}{2 \mu}\right) .
\end{aligned}
$$

Due to the fact, that the real part of $j$ is $\Re(j)<\frac{1}{2}$, the only acceptable momentum is the norm $n=m_{0}=1$. The higher momenta diverge for infinite boundaries. The values, that are found by the formula (55) for higher momenta, are analytical continuations only, e. g. the first momentum $m_{1}$ by formula (55) yields

$$
m_{1}=\mu-\sigma,
$$

which is obviously nonsense, because with norm $n=$ $m_{0}=1$ the first momentum is the expectation value, which is a generalized center of gravity and therefore never can be outside the definition range $x \geq \mu$ of the corresponding function (50). With negative $\sigma$ however, the momentum integral (54) diverges even for the norm $n=m_{0}$.

\subsection{Momenta with momentum generating function in infinite boundaries}

In order to take into account the quality gate, the calculation of the momenta is done using the momentum generating function, which is of the form

$$
g(t)=\int_{a}^{b} e^{t x} f(x) \mathrm{d} x .
$$

For the momenta follows

$$
m_{i}=\left.\frac{\partial^{i}}{\partial t^{i}}(g(t))\right|_{t \rightarrow 0} .
$$

The application to the Lévy distribution (50) for the real part $\mathfrak{R}(\sigma)>0$ and $\mathfrak{R}(t)<0$ leads to

$$
\begin{aligned}
g(t) & =\int_{\mu}^{\infty} \frac{\exp \left(t x-\frac{\sigma}{2(x-\mu)}\right) \sqrt{\sigma}}{\sqrt{2 \pi}(x-\mu)^{\frac{3}{2}}} \mathrm{~d} x \\
& =\int_{0}^{\infty} \frac{\exp \left(t(\mu+\xi)-\frac{\sigma}{2 \xi}\right) \sqrt{\sigma}}{\sqrt{2 \pi} \xi^{\frac{3}{2}}} \mathrm{~d} \xi \\
& =\exp (\mu t-\mathrm{i} \sqrt{2 t \sigma})
\end{aligned}
$$

The case $t \rightarrow 0$ can be used by a limit discussion, where needed. Therefore then the norm $n$ is

$$
n \rightarrow m_{0}=\exp (0)=1 .
$$

The first momentum however diverges by $t \rightarrow 0$ :

$$
\begin{aligned}
& \mu \rightarrow m_{1}= \\
& \left.\exp (\mu t-\mathrm{i} \sqrt{2 t \sigma})\left(\mu-\frac{\mathrm{i} \sqrt{\sigma}}{\sqrt{2 t}}\right)\right|_{t \rightarrow 0}
\end{aligned}
$$

\subsection{Special case of Lévy distribution and some properties}

For the special case of $\mu=0$, the Lévy distribution is of the form

$$
f(x)=\frac{\exp \left(-\frac{\sigma}{2 x}\right) \sqrt{\sigma}}{\sqrt{2 \pi} x^{\frac{3}{2}}} .
$$

With the Lévy distribution (50), the result is valid for the real part $\mathfrak{R}(\sigma)>0$ and $\mathfrak{R}(i)<\frac{1}{2}$, as before. The general result

$$
\begin{aligned}
m_{i} & =2 \mathcal{M}_{x}^{i-\frac{1}{2}}\left(\frac{\exp \left(-\frac{\sigma}{2 x}\right) \sqrt{\sigma}}{\sqrt{2 \pi}}\right) \\
& =2 \int_{0}^{\infty} x^{i-\frac{1}{2}-1} \frac{\exp \left(-\frac{\sigma}{2 x}\right) \sqrt{\sigma}}{2 \pi} d x \\
& =\frac{2^{-i} \sigma^{i} \Gamma\left(\frac{1}{2}-i\right)}{\sqrt{\pi}}
\end{aligned}
$$

can be used for $i=0$ only and furthermore yields $n \rightarrow m_{0}=1$.

The first momentum does not exist. Due to the fact that all momenta like variance, asymmetry are normalized central momenta they do not exist, too.

Here, $\mathcal{M}_{x}^{z}(f(x))$ indicates the Mellin transformation [33] of the function $f(x)$ from $x$ to $z$.

Concerning the asymptotes of the Lévy distribution is noted, that the function

$$
f(x)=\frac{\exp \left(-\frac{\sigma}{2(x-\mu)}\right) \sqrt{\sigma}}{\sqrt{2 \pi}(x-\mu)^{\frac{3}{2}}}
$$


can be described by the asymptote

$$
a(x)=\frac{\sqrt{\sigma}}{\sqrt{2 \pi}(x-\mu)^{\frac{3}{2}}},
$$

since

$$
\begin{aligned}
& \lim _{x \rightarrow \infty}|f(x)-a(x)|= \\
= & \lim _{x \rightarrow \infty}\left|\frac{\exp \left(-\frac{\sigma}{2(x-\mu)}\right) \sqrt{\sigma}}{\sqrt{2 \pi}(x-\mu)^{\frac{3}{2}}}-\frac{\sqrt{\sigma}}{\sqrt{2 \pi}(x-\mu)^{\frac{3}{2}}}\right| \\
= & 0 .
\end{aligned}
$$

\subsection{Discussion of parameters for the second kind of Lévy's distribution}

The second version (51) of the Lévy distribution is a more general one and of the form

$$
f(x)=\frac{\sigma^{k}}{\Gamma(k)} \frac{\exp \left(-\frac{\sigma}{(x-\mu)}\right)}{(x-\mu)^{1+k}} .
$$

Here, three parameters are included. A variation of $k$ rescales the curve (figure (Fig. 3)) and has to be positive.

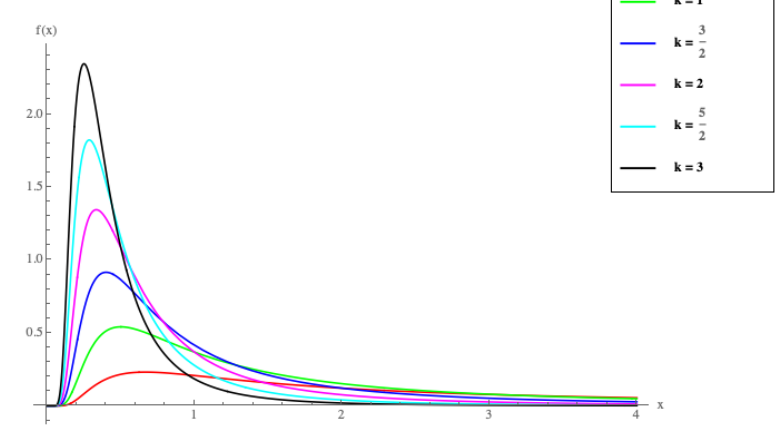

Figure 3:

The Lévy distribution (51) with fixed $\sigma, \mu$ and varying $k$.

According to [31], this parameter correlates with the fractional order of a diffusion equation. The second parameter $\mu \in \mathbb{R}$ generates a translation along the $x$-axis (figure (Fig. 4)), and the changing of $\sigma$ also leads to a rescaling (figure (Fig. 5)). This parameter has to be positive, too. Furthermore from (51) can be seen, that at $x=\mu$ the function owns a singularity for $k \geq 0$.

In order to discuss the domain, $x \geq \mu$ is to be set, even if the function (51) might be defined for some cases of $k$ for a larger space. However, the norm $n=$ $m_{0}=1$ must be fulfilled for all parameters $k, \mu$ and $\sigma$.

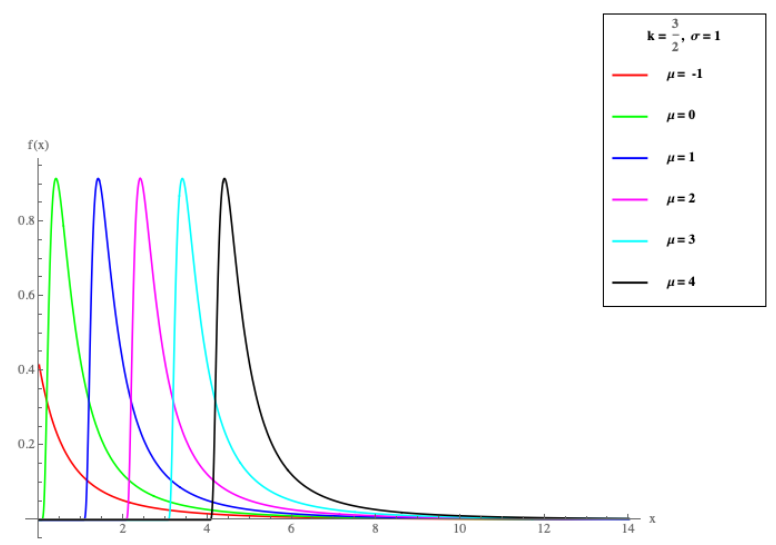

Figure 4:

The Lévy distribution (51) with fixed $k, \sigma$ and varying $\mu$.

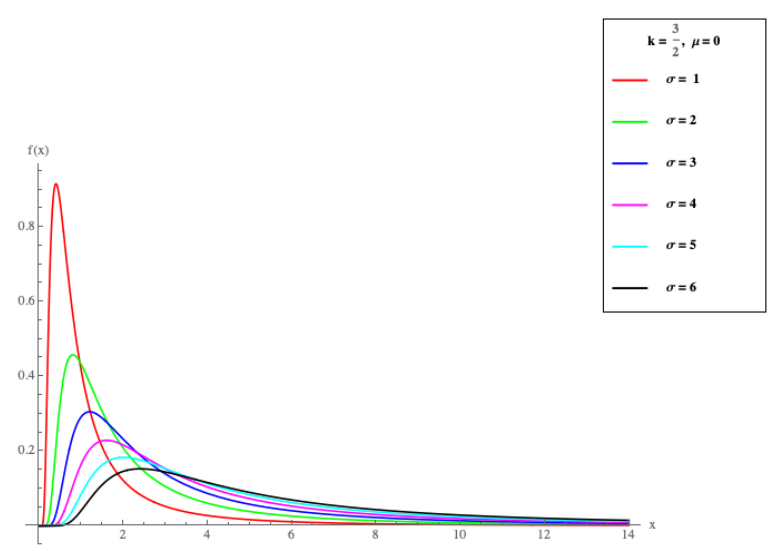

Figure 5:

The Lévy distribution (51) with fixed $k, \mu$ and varying $\sigma$.

The momenta of the Lévy distribution (51) are calculated in the following way:

\subsection{Momenta of second Lévy distribution with momentum integral}

The momentum integral is

$$
m_{i}=\int_{\mu}^{\infty} \frac{x^{i} \exp \left(-\frac{\sigma}{x-\mu}\right) \sigma^{k}}{\Gamma(k)(x-\mu)^{k+1}} \mathrm{~d} x .
$$

After the substitution $x-\mu=\xi, \mathrm{d} x=\mathrm{d} \xi$ follows

$$
m_{i}=\int_{0}^{\infty} \frac{(\xi+\mu)^{i} \exp \left(-\frac{\sigma}{\xi}\right) \sigma^{k}}{\Gamma(k) \xi^{k+1}} \mathrm{~d} \xi .
$$


Using the binomial theorem, this integral can be transformed into

$$
m_{i}=\int_{0}^{\infty} \sum_{j=0}^{i}\left(\begin{array}{l}
i \\
j
\end{array}\right) \xi^{j} \mu^{i-j} \frac{\exp \left(-\frac{\sigma}{\xi}\right) \sigma^{k}}{\Gamma(k) \xi^{k+1}} \mathrm{~d} \xi,
$$

and after swapping summation with integration, the momentum $m_{i}$ can be regarded for the real parts $\mathfrak{R}(\sigma)>0$ and $\mathfrak{R}(k-j)>0$ as

$$
\begin{aligned}
m_{i} & =\sum_{j=0}^{i} \frac{\mu^{i-j} \sigma^{j}\left(\begin{array}{l}
i \\
j
\end{array}\right) \Gamma(k-j)}{\Gamma(k)}= \\
& ={ }_{1} F_{1}\left(-i, 1-k, \frac{\sigma}{\mu}\right) .
\end{aligned}
$$

With this, the following characteristic momenta $m_{i}$ are found for the generalized Lévy distribution with $k>i$ :

$$
\begin{aligned}
& n \rightarrow m_{0}=1, \quad \mu \quad \rightarrow \quad m_{1}=\mu+\frac{\sigma}{k-1}, \\
& \sigma^{2} \rightarrow \frac{\sigma^{2}}{(k-2)(k-1)^{2}}, \quad \sigma^{3} \rightarrow \frac{4 \sigma^{3} k-1}{(k-3)(k-2)(k-1)^{3}} .
\end{aligned}
$$

Here the results for $i \geq 1$ have only formal character due to the fact, that the conditions $k>i, k>0$ and $i>0$ causes singularities for integer $k, i$.

\subsection{Momenta of second Lévy distribution with momentum generating function}

Another way to calculate the momenta, can be done by the momentum generating function:

$$
\begin{aligned}
g(t) & =\frac{\sigma^{k}}{\Gamma(k)} \int_{\mu}^{\infty} \frac{\exp \left(t x-\frac{\sigma}{x-\mu}\right)}{(x-\mu)^{k+1}} \mathrm{~d} x \\
& =\frac{\sigma^{k}}{\Gamma(k)} \int_{0}^{\infty} \frac{\exp \left(t(\mu+\xi)-\frac{\sigma}{\xi}\right)}{\xi^{k+1}} \mathrm{~d} \xi \\
& =\frac{2 \exp (t \mu)(\sqrt{-\sigma t})^{k}}{\Gamma(k)} K_{k}(2 \sqrt{-\sigma t}) .
\end{aligned}
$$

This result, containing the modified Bessel function of the second kind, leads to the same result (68), but not as easy as before.

\section{The Cauchy Distribution}

A second example of a distribution function, which is not less important, is the Cauchy distribution. In literature it is also named after Augustin Cauchy and Hendrik Lorentz as Cauchy-Lorentz distribution or Lorentz distribution. It can be found as Breit Wigner distribution, too. Its form is defined by a function of the type

$$
f\left(x, x_{0}, \gamma\right)=\frac{1}{\pi}\left(\frac{\gamma}{\left(x-x_{0}\right)^{2}+\gamma^{2}}\right)
$$

and arises similarly in the family of curves of third order as "Versiera di Agnesi" (see [4]) and also concerning a Fourier cosine transform ${ }^{C} \mathcal{F}_{t}^{\omega}$ of a function $f(t)=\exp (-\alpha|t|) \cos \left(\omega_{0} t\right)($ see [4] $)$

${ }^{C} \mathcal{F}_{t}^{\omega}\{f(t)\} \sim \frac{\alpha}{\alpha^{2}+\left(\omega-\omega_{o}\right)^{2}}+\frac{\alpha}{\alpha^{2}+\left(\omega+\omega_{o}\right)^{2}}$.

Physically, such kind of functions appear as cross section of resonant nuclear scattering as form ([34])

$$
\sigma(E) \sim \frac{\left(\frac{\Gamma}{2}\right)^{2}}{\left(E-E_{0}\right)^{2}+\left(\frac{\Gamma}{2}\right)^{2}},
$$

as energy distribution for transient spectra of particles, and can be understood as resonance curves in physics of particles, or as driven harmonic oscillator.

In the first part the fundamental solution was determined for the Cauchy problem (37). By setting $\alpha \rightarrow 1$ the Cauchy distribution (45) could be obtained. The momenta of this function (45) are now determined according to the time-fractional diffusion equation, again to obtain information about the quality of such a diffusion process.

\subsection{Parameters and properties of Cauchy distribution}

In this paper, the Cauchy distribution is considered in the form

$$
f(x, \mu, \sigma)=\frac{\sigma}{\pi} \frac{1}{(x-\mu)^{2}+\sigma^{2}},
$$

where $\mu$ and $\sigma$ are parameters. By setting $\mu=0, \sigma=$ $D t$ we arrive at (45). For this function the following limits are valid:

$$
\begin{aligned}
& \lim _{x \rightarrow \infty} \frac{\sigma}{\pi} \frac{1}{(x-\mu)^{2}+\sigma^{2}}=0, \\
& \lim _{x \rightarrow-\infty} \frac{\sigma}{\pi} \frac{1}{(x-\mu)^{2}+\sigma^{2}}=0 .
\end{aligned}
$$

As can be seen in figure (Fig. 6), the variation of the parameter $\mu$ with $\sigma=1$ leads to a translation of the maximum along the $x$-axis. Changes of the parameter $\sigma$ with $\mu=1$ lead to different scaling (see figure (Fig. 7)). Due to the fact, that the function values are related with probabilities, there is a need of $\sigma>0$.

Concerning the asymptotes, the term

$$
\begin{aligned}
& \lim _{x \rightarrow \pm \infty}\left|\frac{\sigma}{\pi\left((x-\mu)^{2}+\sigma^{2}\right)}-\frac{\sigma}{\pi(x-\mu)^{2}}\right| \\
= & \frac{\sigma}{\pi} \lim _{x \rightarrow \pm \infty}\left|\frac{\sigma^{2}}{\left((x-\mu)^{2}+\sigma^{2}\right)(x-\mu)^{2}}\right| \\
= & 0
\end{aligned}
$$




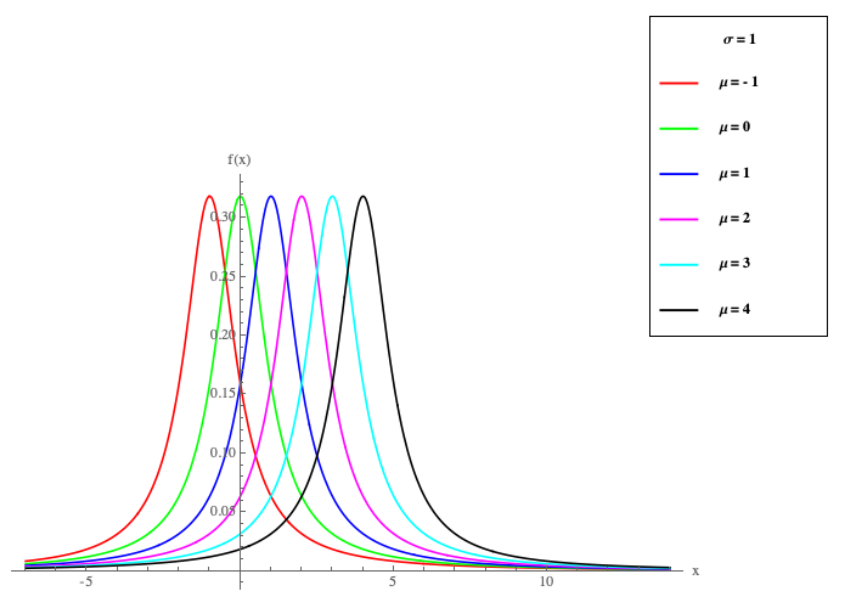

Figure 6:

The Cauchy distribution (70) with fixed $\sigma$ and varying $\mu$.

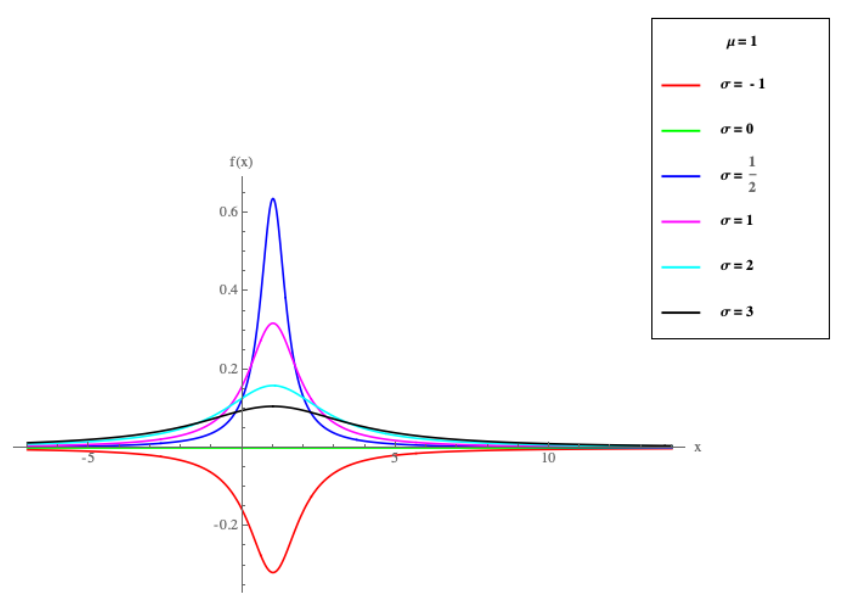

Figure 7:

The Cauchy distribution (70) with fixed $\mu$ and varying $\sigma$.

leads to the asymptote

$$
a(x)=\frac{\sigma}{\pi(x-\mu)^{2}}
$$

of the Cauchy distribution.

\subsection{Momenta of Cauchy's distribution by momentum integral}

Now, for this distribution the momenta are calculated. The related integrals

$$
m_{i}=\int_{-\infty}^{\infty} x^{i} \frac{\sigma}{\pi} \frac{1}{(x-\mu)^{2}+\sigma^{2}} \mathrm{~d} x
$$

have to be calculated according to definition 2. In order to avoid problems, first a primitive for the mo- menta is determined. Starting with the substitution $x-\mu=\xi, \mathrm{d} x=\mathrm{d} \xi$, this leads to

$$
m_{i}=\int_{-\infty}^{\infty}(\xi+\mu)^{i} \frac{\sigma}{\pi} \frac{1}{\xi^{2}+\sigma^{2}} .
$$

The binomial theorem is applied again, and integration and summation are swapped. Thereby results

$$
m_{i}=\frac{\sigma}{\pi} \sum_{j=0}^{i}\left(\begin{array}{l}
i \\
j
\end{array}\right) \mu^{i-j} \int_{-\infty}^{\infty} \frac{\xi^{j}}{\xi^{2}+\sigma^{2}} \mathrm{~d} \xi
$$

Now, the integrand is symmetrical for even $j$ and antisymmetrical for odd $j$. Thus, the odd $j$ can be omitted by $j \rightarrow 2 j$ and $i \rightarrow\left[\frac{i}{2}\right]$, where the Gaussian bracket function $[n]$ gives an integer $[n] \leq n$. By this results $j=0$ :

$$
\begin{aligned}
& m_{i}= \\
& \sum_{j=0}^{\left[\frac{i}{2}\right]}\left(\begin{array}{c}
i \\
2 j
\end{array}\right) \mu^{i-2 j} \sigma^{2 j} \Gamma\left(\frac{1}{2}-j\right) \Gamma\left(\frac{1}{2}+j\right) .
\end{aligned}
$$

The momenta diverge for $j>0$. Therefore, the norm $n=1$ and the expectation value $\mu \rightarrow \mu$ can be used only. The formal variance $\sigma^{2} \rightarrow-\sigma^{2}$ is negative, which has no meaning.

\subsection{Momemta of Cauch's distribution by momentum generating function}

In order to fulfill the quality gate, being described above, the way using the momentum generating functions leads to

$$
\begin{aligned}
m_{i} & =\left.\frac{\partial^{i}}{\partial t^{i}}\left[\int_{-\infty}^{\infty} e^{t x} f(x) \mathrm{d} x\right]\right|_{t \rightarrow 0} \\
& =\left.\frac{\partial^{i}}{\partial t^{i}}\left[\int_{-\infty}^{\infty} e^{t x} \frac{\sigma}{\pi} \frac{1}{(x-\mu)^{2}+\sigma^{2}} \mathrm{~d} x\right]\right|_{t \rightarrow 0}
\end{aligned}
$$

This integral leads to the exponential integral function and causes more complicate results than (76).

\section{Discussion and Conclusion}

In the previous sections the statistical momenta of various fractional diffusion equations are considered.

For the time fractional equation the general expression for the momenta of $m$-th order was found in accordance to the quality gate. This is of the form

$$
M(m)=\frac{a^{\frac{m}{2}} t^{\frac{m \alpha}{2}} \Gamma(1+m)}{\Gamma\left(1+\frac{m \alpha}{2}\right)} .
$$


From this the significant momenta like expectation value, variance and asymmetry are determined. For the norm the result is

$$
n=M(0)=1 .
$$

The expectation value is usually represented as the first normalized momentum. One finds

$$
\mu=\frac{M(0)}{M(1)}=\frac{\sqrt{a} t^{\frac{\alpha}{2}}}{\Gamma\left(1+\frac{\alpha}{2}\right)}
$$

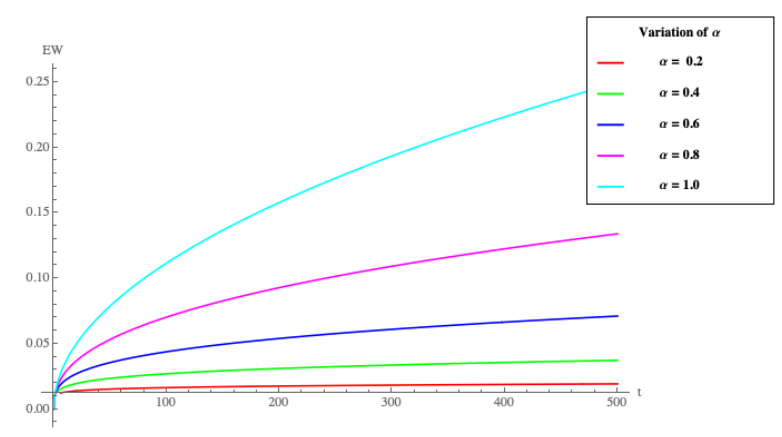

Figure 8:

Expectation value of the solution (11) of time-fractional diffusion equation with varying $\alpha$ and $a=98 * 10^{-6}$.

If one looks at the graphical representation for different values of $\alpha$ one finds different root functions whose steepness increases with increasing $\alpha$.

Furthermore, it can be seen that the functions for the expectation value are monotonically increasing with time. Howevery, since this quantity can be interpreted as a thermal center, the question of model alternatives is raised here, because in experiments it does not move only in one direction.

The variance is from statistical point of view defined as the central normalized second momentum. It is

$$
\begin{aligned}
\sigma^{2} & =\frac{M(2)}{M(0)}-\frac{M(1)^{2}}{M(0)^{2}} \\
& =a t^{\alpha}\left(-\frac{1}{\Gamma\left(1+\frac{\alpha}{2}\right)^{2}}+\frac{2}{\Gamma(1+\alpha)}\right)
\end{aligned}
$$

Due to the fact that $\alpha$ is in $[0,1]$ we have root functions for this quantity, too. These are also monotonously increasing.

Furthermore it should be pointed out, that in literature the variance is equated with the second momentum (see e. g. [15, 35, 36]. Using the definition from statistics, according which the variance is the second normalized central momentum, other proportionality constants result, which stand ahead of the time power.

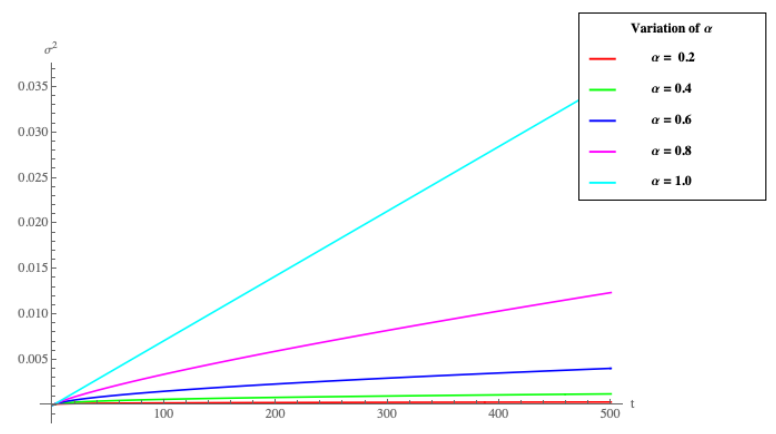

Figure 9:

The Variance of the solution (11) of time-fractional diffusion equation with varying $\alpha$ and $a=98 * 10^{-6}$

The asymmetry is the central normalized third momentum with

$$
\begin{aligned}
\sigma^{3} & =\frac{M(3)}{M(0)}-3 \frac{M(2) M(1)}{M(0)^{2}}+2 \frac{M(1)^{3}}{M(0)^{3}} \\
= & 2 a^{\frac{3}{2}} t^{\frac{3 \alpha}{2}}\left(\frac{1}{\Gamma\left(1+\frac{\alpha}{2}\right)^{3}}-\frac{3}{\Gamma\left(1+\frac{\alpha}{2}\right) \Gamma(1+\alpha)}+\right. \\
& \left.\frac{3}{\Gamma\left(1+\frac{3 \alpha}{2}\right)}\right) .
\end{aligned}
$$

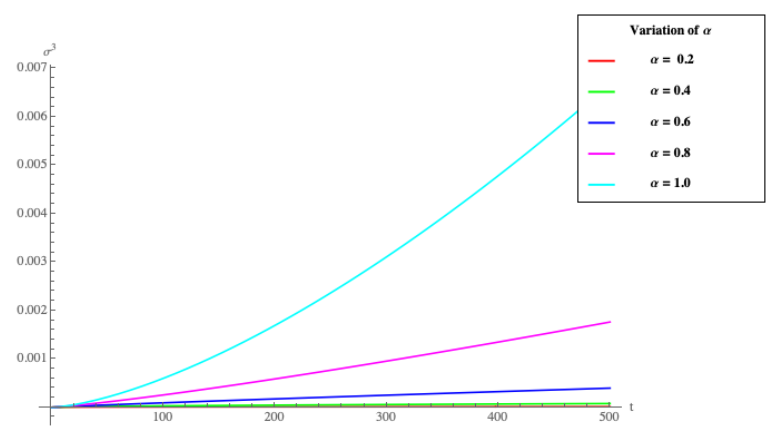

Figure 10:

The Asymmetry of the solution (11) of time-fractional diffusion equation with varying $\alpha$ and $a=98 * 10^{-6}$.

The arithmetic expression is again proportional to a power of the time $t$, whereby $\alpha$ is included in the exponent. The asymmetry also represents a monotonous function in $t$ for $\alpha \in[0,1]$.

The consideration of all momenta makes clear that model extensions and alternatives are needed to describe real experiments with this theory, because measurements on heat conduction will show that the statistical momenta expectation value, variance and asymmetry are anything but monotonous functions.

Furthermore, at this point the question must be asked whether it is at all permissible to compare a 
theory in infinite boundaries with experimental data in finite boundaries.

The second equation which has been investigated had a space-fractional derivative which has been described using the Riesz operator. The result was the Lévy - and Cauchy distribution for special choices of the derivative parameter $\alpha$. For both distributions the momenta in infinite boundaries were determined. Here, however, it was shown that some of them did not exist at all. There is also the discrepancy that theoretical momenta do not exist but measured ones can be determined.

This suggests in a first step the extension of the theory to momenta in finite boundaries. Hereby the existence of the momenta for the solution of the space-fractional differential equation (36), which are Lévy distribution and Cauchy distribution, is established.

Furthermore, theory and experiment have to be combined. Another forthcoming paper discusses this on the basis of a heat conduction experiment.

\section{References:}

[1] Einstein, A., Über die von der molekularkinetischen Theorie der Wärme geforderte Bewegung von in ruhenden Flüssigkeiten suspendierten Teilchen (About the movement of particles suspended in quiescent fluids required by the molecular-kinetic theory of heat), Ann. d. Phys. , Vol. 322, Issue 8, 1905, pp. 549-560.

[2] Smoluchowski, M. V., Zur kinetischen Theory der Brownschen Molekularbewegung und der Suspension (On the kinetic theory of Brownian motion and the suspension), Ann. d. Phys., Vol. 326, Issue 14, 1906, pp. 756-780.

[3] Smoluchowski, M. V., Über Brownsche Molekularbewegung unter Einwirkung äußerer Kräfte und deren Zusammenhang mit der verallgemeinerten Diffusionsgleichung (About Brownian motion under the influence of external forces and their relation to the generalized diffusion equation), Ann. d. Phys., Vol. 353, Issue 24, 1916, pp. 1103-1112.

[4] Bronstein, J. N.; Semendjajew, K. A., Taschenbuch der Mathematik (Handbook of Mathematics), Harri Deutsch GmbH, 1985

[5] Volkmann, J.; Suedland, N, The Variance Theorem for Infinite Boundaries: Theory and Application, Technical Report, Researchgate, February 2018

[6] Bouchaud, J. Ph.; Georges, A., Anomalous Diffusion in Disordered Media: Statistical Mechan- ics, Models and Physical Applications, Physics Reports, 195, 1990, 127 - 293

[7] Klafter, J.; Schlesinger, M. F.; Zumofen, G., Beyond Brownian Motion, Physics Today, 49, 1996, 33 - 39

[8] Metzler, R.; Klafter, J., The Random Walk's Guide to Anomalous Diffusion: a Fractional Dynamics Approach, Physics Reports, 339, 2000, 1 - 77

[9] Sokolov, I. M.; Klafter, J., Blumen, A., Fractional Kinetics, Physics Today, 55, 2002, 48 54

[10] Zaslavsky, G. M., Chaos, Fractional Kinetics and Anomalous Transport, Physics Reports, $371,2002,461-580$

[11] Metzler, R.; Klafter, J., The Restaurant at the End of the Random Walk: Recent Developments in the Description of Anomalous Transport by Fractional Dynamics, J. Math. Phys: Math. Gen., 37, 2004, R161 - R208

[12] Chechkin, A. V.; Gonchar, V. Y.; Klafter, J.; Metzler, R., Fundamentals of Lévy Flight Processes, Advances in Chemical Physics , 133B, 2006, 439 - 496

[13] West, B. J.; Grigolini, P.; Metzler. R.; Nonnenmacher, T. F., Fractional diffusion and Lévy stable processes, Phys. Rev. E, 55, 1997, 99 - 106

[14] Südland, N.; Volz, Ch.; Nonnenmacher, T. F., A Fractional Calculus Approach to Adsorbate Dynamics in Nanoporous Materials, Fractals in Biology and Medicine, Vol. III, Birkhäuser, 2002, $325-332$

[15] Metzler. R.; Chechkin, A. V.; Klafter, J., Lévy Statistics and Anomalous Transport: Lévy Flights and Subdiffusion, arXiv:0706.3553v1, [cond-mat.stat-mech], June 2007

[16] Chechkin, A. V.; Metzler, R.; Klafter, J.; Gonchar, V. Yu., Introduction to the theory of Lévy Flights, in: Klages, R.; Radons, G.; Sokolov, I. M., Anomalous Transport, Wiley-VCH, 2008

[17] Volkmann, J.; Suedland, N, Transport Phenomena and the Variance Theorem for Infinite Boundaries, Proceedings of the Conference Differential Equations and Related Problems, Interdisciplinary Conference, Sterlitamak, 2018, 166 $-172$

[18] King, J.,The Bible, Authorized Version, Deut, 19:15, Oxford University Press, 1994 
[19] Mathai, A. M.; Saxena, R. K.; Hausbold, H. J., The H-function. Theory and Application, Springer, 2010

[20] Grimmett, G. , Stirzaker, D., Probability and Random Processes, Oxford University Press, 2009

[21] Fisz, M., Wahrscheinlichkeitsrechnung und Mathematische Statistik (Probability Theory and Mathematical Statistics), VEB,Berlin 1989

[22] www.dartmouth.edu/ chance/teaching_aids/ books_articles/probability_book/Chapter10.pdf

[23] Chen, Wen, A speculative study of $\frac{2}{3}$-order fractional Laplacian modeling of turbulence: Some thoughts and conjectures, Chaos 16, 2006, 023126

[24] Weron, K.; Jurlewicz, A., Two Forms of Selfsimilarity as a Fundamental Feature of the Power Law Dielectric Response, J. Phys. A: Math. Gen., 26, 1993, 395 - 410

[25] Bouchaud, J. Ph.; Vincent, E.; Hammann, J., Towards an Experimental Determination of the Number of Metastable States in Spin-glasses, $J$. Phys. I France, 4, 1994, 139 - 146

[26] Schlesinger, M. F.; Zaslavsky, G. M.; Friesch, U., Lévy Flights and Related Topics in Physics, Proceedings of the international Workshop held at Nice, France, June $27^{t h}-30^{t h} 1994$, Springer 1995

[27] Boldyrev, St.; Gwin, C. R., Lévy Model for Interstellar Scintillations, Phys. Rev. Lett., Vol 91, Issue 13, 131101 or arXiv: astro-ph/0309644v1, September $23^{\text {rd }} 2003$

[28] Sibatov, R.; Uchaikin, V. V., Truncated Lévy Statistics for Transport in Disordered Semioconductors, Proceedings of the $3^{\text {rd }}$ Conference on Nonlinear Science and Complexity, Ankara, July $28^{\text {th }}-31^{\text {st }}, 2010$

[29] Sokolov, I. M., Lévy Flights from a Continuoustime Process, Phys. Rev. E, Vol. 63, Issue
1, 011104, 2000; arXiv:cond-mat(00074961v1 (July $30^{\text {th }} 2000$ )

[30] Cybierc, B.; Sokolov, I. M.; Chechkin, A. V, Stationary States under Anomalous Dynamics, Proceedings of the $3^{\text {rd }}$ Conference on Nonlinear Science and Complexity, Ankara (July $28^{\text {th }}-$ $31^{\text {st }}, 2010$

[31] Nonnenmacher, T. F., Fractional Integral and Differential Equations for a Class of Lévy-type Probability densities, J. Phys. A: Math. Gen., 23, 1990, L697S - L700S

[32] Südland, N., Anwendungen der Foxschen HFunktion (Application of Fox's H-Function), Diplom Thesis, Ulm 1997

[33] Oberhettinger, F., Tables of Mellin Transforms, Springer, Berlin 1974

[34] Bergmann, L., Schaefer, C., Raith, W., Lehrbuch der Experimentalphysik (Handbook of Experimetnal Physics), Vol. 4 Teilchen (Particles), Walter de Gruyter, Berlin etc. 1992

[35] Rida, S. Z.; El-Sayed, A. M. A.; Arafa, A. A. M., On the solution of time-fractional reactiondiffusion equations, Commun. Nonlinear Sci. Numer. Simulat, 15, 2010, 3847 - 3854

[36] Evangelista, L. R.; Lenzi, E. K., Fractional Diffusion Equations and Anomalous Diffusion, Cambridge University Press, 2018

\section{Contribution of individual authors to the creation of a scientific article (ghostwriting policy)}

Joerg Volkmann carried out the calculations. Norbert Südland has developed and implemented the Fractional Calculus Algorithms in Mathematica.

Nail Migranov has developed Fractional Calculus Algorithms, too and has done editorial work

\section{Creative Commons Attribution License 4.0 (Attribution 4.0 International, CC BY 4.0)}

This article is published under the terms of the Creative Commons Attribution License 4.0

https://creativecommons.org/licenses/by/4.0/deed.en_US 